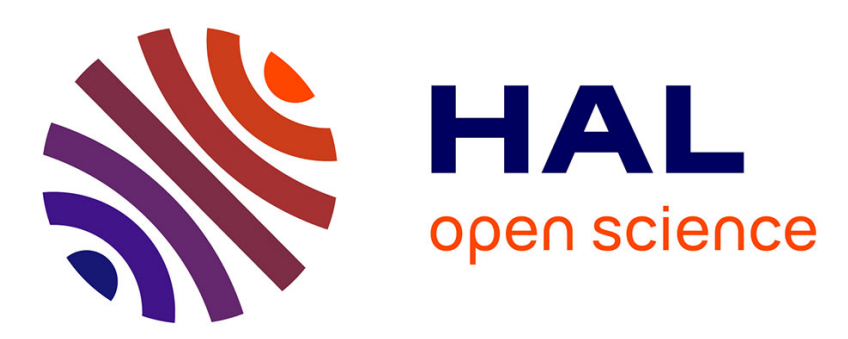

\title{
Wireless Network Simulators Relevance Compared to a Real Testbed in Outdoor and Indoor Environments
}

\author{
Abderrezak Rachedi, Stéphane Lohier, Sylvain Cherrier, Ismail Salhi
}

\section{To cite this version:}

Abderrezak Rachedi, Stéphane Lohier, Sylvain Cherrier, Ismail Salhi. Wireless Network Simulators Relevance Compared to a Real Testbed in Outdoor and Indoor Environments. 6th International Wireless Communications and Mobile Computing Conference (IWCMC'10), Jul 2010, France. pp.346350. hal-00620343

\section{HAL Id: hal-00620343 https://hal.science/hal-00620343}

Submitted on 6 Apr 2012

HAL is a multi-disciplinary open access archive for the deposit and dissemination of scientific research documents, whether they are published or not. The documents may come from teaching and research institutions in France or abroad, or from public or private research centers.
L'archive ouverte pluridisciplinaire HAL, est destinée au dépôt et à la diffusion de documents scientifiques de niveau recherche, publiés ou non, émanant des établissements d'enseignement et de recherche français ou étrangers, des laboratoires publics ou privés. 


\title{
Wireless Network Simulators Relevance Compared to a Real Testbed in Outdoor and Indoor Environments
}

\author{
Abderrezak Rachedi, Stéphane Lohier, Sylvain Cherrier, Ismail Salhi \\ Computer Science Laboratory (LIGM) \\ University of Paris-Est Marne-la-Vallée (UPEMLV) \\ Champs sur Marne, France \\ \{rachedi, lohier, cherrier, salhi\}@univ-mlv.fr
}

\begin{abstract}
In this paper, we propose a comparative study between results generated by a real 802.11 testbed in different outdoor and indoor environments and 3 usual network simulators (NS2, QualNet and OPNET). The goal of this study is to evaluate the relevance of the low layers recently implemented in these simulators. The motivation of this paper is to provide a guide to researchers to choose and parameterize a simulator according to a selected context. The study shows that the simulation results can be rather close to the experimental results. However, they are very dependent on the tuning of the physical layer parameters and the selected propagation models.
\end{abstract}

Keywords: Wireless Networks, 802.11, Network Simulators, Testbed, Propagation Models

\section{INTRODUCTION}

The design and the implementation of new protocols or models for wireless multi-hop networks such as MANET or WSNs are mainly based on simulation in order to test and validate the proposed solutions. The main reasons are the cost and the complexity of a real implementation in a testbed, especially when the network is dense and the scaling factor has to be tested. In all cases, the simulations allow a fast performance comparison between different protocols/models with different scenarios and for many times. However, in the real world tests (testbeds) and particularly in the wireless communication case, the impact of some parameters such as: the type of environment (indoor/outdoor), the terminal location and movement are very important and can influence the performance evaluation of protocols or models. In order to determine the relevance of the wireless network simulators, we have to answer both following questions: 1) Does the implementation of the lower layers (particularly the physical layer) in the network simulators reflect the reality? In other words, does it give results close to the reality? 2) if the implementation does not reflect the reality, what is the relevance of the obtained results in the case of a dense multihop network, knowing that the transmission model between two nodes is not realistic?

Thus, our motivation for this work is based on three aspects: the first is related to the important number of researchers who use the simulator networks to validate their solutions. For example, according to Kurkowski et al. study, more than $75.5 \%$ of the published papers for MobiHoc symposium used simulation to validate the proposed solutions [1]. The second aspect is related to the existence of significant divergences in the obtained simulation results based on several popular simulators such as OPNET [3], NS-2 [4] and GloMoSim [5]. These divergences are illustrated by Cavin et al. [2] study. The third and final aspect of our motivation is to give an explanation of these differences between simulation results by using and comparing them to a real testbed.

Finally, the two main contributions of this paper are: 1) highlight the differences at the lower layers between the simulators and reality; 2) point out the sensitive parameters and models which can influence the simulation results and lead to an important difference between the simulation and the testbed results. In order to achieve these goals, we choose two scenarios (indoor and outdoor) with a focus on the transmission between two nodes and three major network simulators: NS2, OPNET and QualNet.

This paper is organized as follows: in section 2, we present the summarization of the existing works related to network simulators and the experimentation results. Section 3 presents the selected scenarios and the results for the testbed. The fourth section is dedicated to the results obtained with the simulators and their analysis, compared to the testbed. Finally, section 5 concludes the paper and presents our future works.

\section{RELATED WORK}

In literature many works deal with the comparison between different network simulators. For instance, the accuracy study of different MANETs simulators (OPNET, NS2, GloMoSim) is presented by Cavin et al. [9]. The obtained results from the three network simulators illustrate the significant divergences which are due to the mismatching of the modelling of each simulator. However, no comparison with real wireless network testbed is presented. Another study presented by Kargl and Schoch [10] consists in comparing network simulators JiST/SWANS and NS2. This study shows that NS2 needs a more important memory compared to SWANS (NS2 consumes $300 \mathrm{MB}$ with the scenario of 150 nodes, but SWANS does not require more than 10MB).

Therefore, the results divergences between different network simulators lead us to compare network simulators in terms of accuracy with the testbed as reference. That's why Lucio et al. [8] proposed a comparison study between the simulators NS2 and OPNET and the testbed of wired networks. The goal of 
this study is to present the simulator which gives the result closest to reality under the given conditions. In the case of the CBR traffic, Ns2 gives more accurate results than OPNET Modeler and in the case of FTP traffic OPNET performed more closely to the testbed results. This study is limited to wired networks.

In other works, the goal is to validate into simulators the implementation of MAC layer protocols such as IEEE802.11g and IEEE802.11e. For example, the study presented by Bredel and Bergner [12] consists in implementing the IEEE802.11g under OMNET++ simulator. The conclusion of this study is that OMENT++ performs quite well in the case of long observation times compared to the testbed results. However, they assume that the physical medium is free of interference from external sources and the fading effects are negligible. Furthermore, the indoor environment is not taken into account which makes the comparison not close to the reality. Garrido et al. [13] have proposed a comparative implementation of the IEEE 802.11e technology with both simulators NS2 and OPNET. The obtained results point out some important differences between both simulators. However, the testbed is not taken into account.

Other studies focus on the indoor environment, particularly the propagation models used by the simulators in order to justify the divergences between the different simulator networks. Stepanov et al. [6] propose to integrate a more accurate radio propagation model into the simulation tool. The proposed model is based on ray tracing and considers geographic data of the simulation area. In order to test this propagation model, they used a commercial implementation of the intelligent ray tracing model called WinROP [7]. The drawbacks of this work are: first, no experimentation or testbed is done to compare with the simulation results. Secondly, the data set generated for the propagation model is from WinPROP which is a commercial tool.

Another factor affecting the accuracy of the simulation results is the background noise. The noise models implemented in the network simulators such as: NS2, OPNET and Glomosim are too simplistic and do not reflect the real network conditions. That's why Su and Boppana [11] proposed the measurement of background noise for MANET using a testbed and they illustrate the potential noise modelling by using the generalized extended value (GEV) and the discrete-time Markov chain. However, no impact evaluation of the proposed noise model is presented. In addition, no new or more realistic propagation model is presented.

Unlike the existing works, in this paper we focus on the two main environments (outdoor and indoor) and we present and compare the obtained results from three major network simulators (NS2, OPNET, QualNet), without modifying them, with testbed results. The added value of our study is to present and analyse the simulation results and the testbed results in order to point out the divergence between them and the causes of these divergences.

\section{TESTBED}

\section{A. Context}

The environment has an important impact on the wireless communications. Indoor environment is more spread in reality, for example using wireless access in companies, offices, airports, and hotspots. Outdoor environment represents the free space area without physical obstacle like for an emergency deployment in a rural environment what is much less frequent. Let us note that a lot of performance simulation studies on dense wireless networks use the free space model which is not very realistic.

For this study the indoor environment is our lab, made up of $15-30 \mathrm{~m}^{2}$ offices distributed along a $50 \mathrm{~m}$ corridor. This is a usual office context. Like for access point deployment, the nodes are located in the corridor to take into account the fading effects. Other transmissions in the band of $2.4 \mathrm{GHz}$ exist in this area and in the vicinity but we selected a free channel. For the outdoor (free space) environment, we chose a park near our university without obstacles.

For both contexts, we have selected a simple point-to-point scenario with one communication flow (UDP or TCP) and different distances between the sender and the receiver. We used 4 laptops with Netperf [15] and MGEN [14] as network traffics generator.

The embedded wireless devices use the IEEE 802.11g standard which is currently widespread. With this technology, the transmission range in experimentation is limited to almost $35 \mathrm{~m}$. That is why we varied the distance between the sender and the receiver from 5 to 40 meters and we estimated the results for each distance. The selected metrics for this study are the throughput and the signal level in order to evaluate the performance of the transmission and the quality of the channel.

\section{B. Results}

Figure 1 illustrates the average values of the received signal level (in $\mathrm{dBm}$ ) and the throughput measured at the receiver according to the different distances between the sender and the receiver nodes in the case of a TCP flow. Measurements were made in both indoor and outdoor environments with our testbed. In the case of outdoor results and according to the mathematic formulation of free space environment, the signal level should decrease with distance square. The measured signal level is more versatile than expected. It depends on many parameters such as: the characteristics and the orientation of the antennas, the reflexions on the floor, the possible background noise etc. In the case of indoor environment, we expected an important variability of the signal level. Our testbed is located in the corridor of our laboratory and both nodes are in line of sight, but as in every real place, people are moving in the area. The measurements show that the signal level is quite close to the one obtained in outdoor. As the selected channel is free, the transmissions in the vicinity do not cause interferences being able to disturb the signal significantly. We even obtain slightly better values $(+8$ $\mathrm{dBm}$ ) probably due to the close reflections on the walls (corridor effect). 


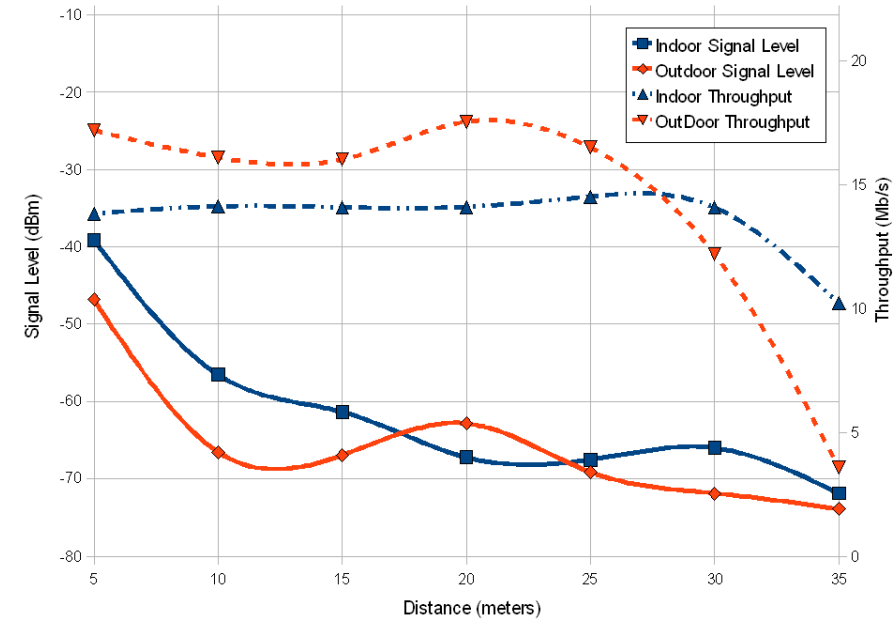

Figure 1. Average Signal Level and Throughput / Distance (testbed)

What is the impact of that signal level's variation on the throughput? As we can see in figure 1, for short distances, the throughput remains constant (real throughput between 14 and $18 \mathrm{Mb} / \mathrm{s}$ corresponding to a $54 \mathrm{Mb} / \mathrm{s}$ Data Rate) even if the signal level decreases. Then, for a distance greater than $25 \mathrm{~m}$, the throughput decreases quickly. We can deduce that the ARF (Auto Rate Fallback) procedure is effective in this case: the 802.11 device tries to maintain a high data rate $(54 \mathrm{Mb} / \mathrm{s})$ until a power threshold is reached (approximately $-70 \mathrm{dBm}$ ). Let us remind that we represent the average values of the signal level and the throughput measured at a certain distance: for $30 \mathrm{~m}$, the level or the throughput can be maximum or null at certain times.

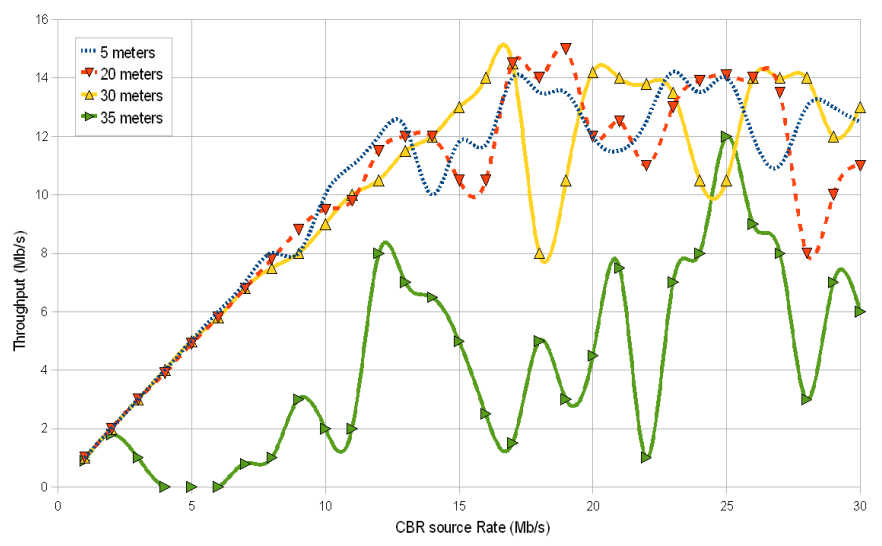

Figure 2. Average Throughput / CBR sources Rate (testbed)

For the second measurement, we use a CBR/UDP source instead of a FTP/TCP source in order to observe the behavior of our connection when the flow increases linearly in an indoor environment. Figure 2 illustrates the average throughput when the rate of the CBR source varies and for different distances between the sender and the receiver. After estimating the throughput with NetPerf and MGEN, we started a sequence of transmissions with the rate of the CBR flow varying from $1 \mathrm{Mb} / \mathrm{s}$ to $30 \mathrm{Mb} / \mathrm{s}$. The throughput is quite stable for a CBR source rate lower than $13 \mathrm{Mb} / \mathrm{s}$. For higher rates, the throughput is highly variable, not only depending on the CBR source. Once again, the 802.11 device tries to preserve a maximum Data Rate but this one is reduced as soon as the signal decreases. This trend is not predictable. However the amplitude of throughput variations remains related to the distance. The green curve shows the throughput variation when we are near the edge of range $(35 \mathrm{~m})$. In that case, the throughput depends more on the signal level which is near the threshold $(-70 \mathrm{dBm})$ than on the CBR source rate.

\section{SIMULATORS}

\section{A. NS-2 [4]}

NS-2 is currently the most popular network simulator: more than $43 \%$ of researchers have been using NS-2 to evaluate their proposed solution [1]. We adapted the rate of the existing IEEE802.11 MAC layer to support the IEEE802.11g current standard. However, a real PHY layer with OFDM multiplexing is not implemented. We used the FreeSpace and TwoRayGround propagation models to simulate the Outdoor environment and the Shadowing model with different parameters to simulate the Indoor environment. To be close to the real testbed, neither background noise nor interference in the same channel is simulated in the vicinity of both nodes. For the Free Space model, the power attenuation of the signals is proportional to $1 / d^{2}$ :

$$
P_{r}=\frac{P_{t} G_{t} G_{r} \lambda^{2}}{(4 \pi d)^{2} L}
$$

$G_{t}$ and $G_{r}$ are the antennas gains, $\lambda$ the wavelength and $L$ the Loss Factor. We choose values of Transmitted Power $\left(P_{t}\right)$ and Capture Threshold $\left(P_{r}\right)$ to obtain the same range as in the real testbed. These reference values are also used for the other propagation models.

For the shadowing model, two important parameters are used to differentiate the environment:

$$
\left[\frac{P_{r}(d)}{P_{r}\left(d_{0}\right)}\right]_{d B}=-10 \beta \log \left(\frac{d}{d_{0}}\right)+X_{d B}
$$

Where $d_{0}$ is a reference distance. $\beta$ is the path loss exponent and is usually empirically determined by field measurement. For example, $\beta=2$ corresponds to free space propagation. When we set a larger value of $\beta$ that means that the number of obstructions is more important and the greater the distance, the faster the decrease of the received signal power. The second parameter $X_{d B}$ is a log-normal random variable and reflects the variation of the received power at certain distance. $X_{d B}$ is thus a Gaussian random variable with zero mean and standard deviation $\sigma_{d B}$ which is called the shadowing deviation. $\sigma_{d B}$ is also obtained by measurement. For example, $\sigma_{d B}=7 \mathrm{~dB}$ corresponds to an obstructed office environment. The simulation parameters are resumed in table 1 .

TABLE 1: SIMULATION PARAMETERS

\begin{tabular}{|l|l|}
\hline Physical Channel & Extended Rate $-802.11 \mathrm{~g}$ \\
\hline Data Rate & $54 \mathrm{Mbps}$ \\
\hline Reception range (Capture Threshold) & $40 \mathrm{~m}$ \\
\hline Detection range (Carrier Sense Threshold) & $80 \mathrm{~m}$ \\
\hline UDP packet size (CBR sources with max. rate) & 1000 Bytes \\
\hline
\end{tabular}


The results are plotted in figure 3. First, we notice that for short distances, the throughput obtained by simulation is always higher, whatever the model is. This is mainly due to the lack of a specific $802.11 \mathrm{~g}$ physical layer.

The Free Space and the Two Ray Ground models give the same results which are not representative. The ARF procedure is not implemented in NS-2 and for these simplistic models, the propagation is maximum or null beyond a certain distance.

The shadowing model offers results closer to reality but the parameters that drive shadowing propagation are difficult to set. The documentation of NS-2 gives typical values of $\beta$ and $\sigma$ (for example: $\beta=2$ and $\sigma=4 \mathrm{~dB}$ to 12 for an outdoor free space environment; $\beta=4$ to 6 and $\sigma=6.8 \mathrm{~dB}$ for an indoor obstructed environment) but the shadowing model is probabilistic and insofar as these parameters have to be determined by field measurement, it is difficult to reflect the reality. Thus, it is possible to adjust the parameters to obtain a curve close to reality (indoor or outdoor) but these adjustments cannot be generalized. In figure 3 , for $\beta=1.8$ and $\sigma=3.5$, the throughput presents the same decrease as for the testbed in outdoor. However, according to the model, these parameters correspond to an "in building, line-of-sight" environment. Moreover, a wrong tuning can lead to false results (see yellow curve in figure 3 ).

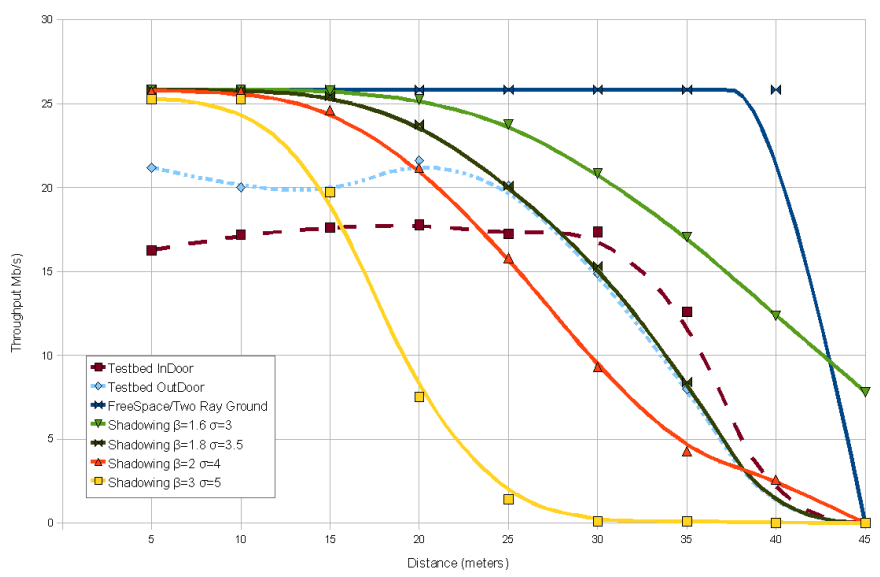

Figure 3. Average Throughput / Distance (Testbed compared to NS-2)

\section{B. QualNet[16]}

In order to perform an efficient and fair comparison between the simulators, we used the same parameters as in NS-2, whether for the PHY or MAC layer as summarized in table 1. Let us note that the implementation of the PHY layer is not complete for the IEEE $802.11 \mathrm{~g}$ standard. Nevertheless, Qualnet is the only simulator offering the possibility of using the ARF procedure. The proposed propagation models are closed to those proposed in NS-2: free space, two ray ground and log-normal shadowing (in this only the deviation parameter is used). Qualnet also proposes Rayleigh fading model, which occurs when there is no line of sight between the source and destination.

Note that Qualnet proposes additional propagation models for different outdoor configurations, like the irregular terrain model, the urban model, the Walfish-Ikegami model, suburban and other terrain feature formats such as Geographic Information System (GIS) and Compact Terrain DataBase (CTDB). As for OPNET, these models are specifics to large outdoor environment and give results very close to the free space or two ray ground models.

Figure 4 shows the results of the Qualnet simulations. The first observation concerns the average values of the throughputs which are close to those obtained in the testbed (indoor and outdoor), comparing to NS-2 and Opnet. We can also see that the ARF enhances considerably the accuracy of the results. As for NS-2, the shadowing model gives the best results for the indoor environment. Here also, the shadowing deviation is adjusted to obtain a curve close to reality $(\sigma=4 \mathrm{~dB})$ and the adjustment is much more precise and effective than for NS.

In the Rayleigh fading model, with a velocity parameter of $1 \mathrm{~m} / \mathrm{s}$ (pedestrian moving), the decreasing is quite linear and does not correspond to the selected real environment.

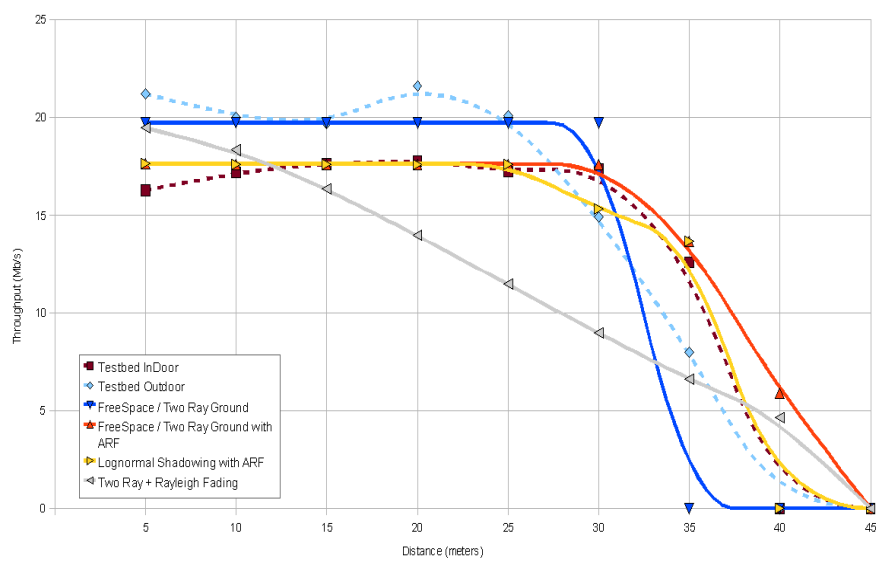

Figure 4. Average Throughput / Distance (Testbed compared to Qualnet)

\section{OPNET}

OPNET Modeler is a network simulator well known in industry. The wireless suite of OPNET integrates $802.11 \mathrm{~g}$ devices (PHY Extended Rate) and offers 5 propagation models intended for outdoor environments (Free Space, Longley-Rice, Hata, CCIR, Walfisch-Ikegami). OPNET Modeler views all wireless channels as Gaussian channels (uniform noise spectral density) and ignores the fading effect. In addition, OPNET uses a fixed value of the pathloss exponent without considering the diversity of the environments. Like for NS-2 and Qualnet the transmitted power is adjusted in the free space model to obtain a $40 \mathrm{~m}$ reception range. The other parameters are the same (see table 1). The results for the free space model are plotted in figure 5. With the same power value, the other propagation models give very nearby results and are thus not represented. They are indeed designed for outdoor environment and thus for transmission powers much higher than those usually used in IEEE 802.11 (greater than $100 \mathrm{~mW}$ ) and for antennas with a range much higher than those present in the laptops (greater than $100 \mathrm{~m}$ ). For example, Hata is a widely used propagation model suitable for predicting RF path loss in an urban environment. This model has a parameter, which specifies the 
relative size and distribution of buildings (large city, small city, suburban, and open areas) and antenna heights are chosen starting from the selected size.

As conclusion, although OPNET integrates 802.11g device and a lot of possible adjustments (noise, loss factor, antenna models...), the propagation models proposed by default do not make it possible to carry out simulations close to reality for short distances, particularly in an indoor environment. It is necessary in this case to add other propagation models like the shadowing type.

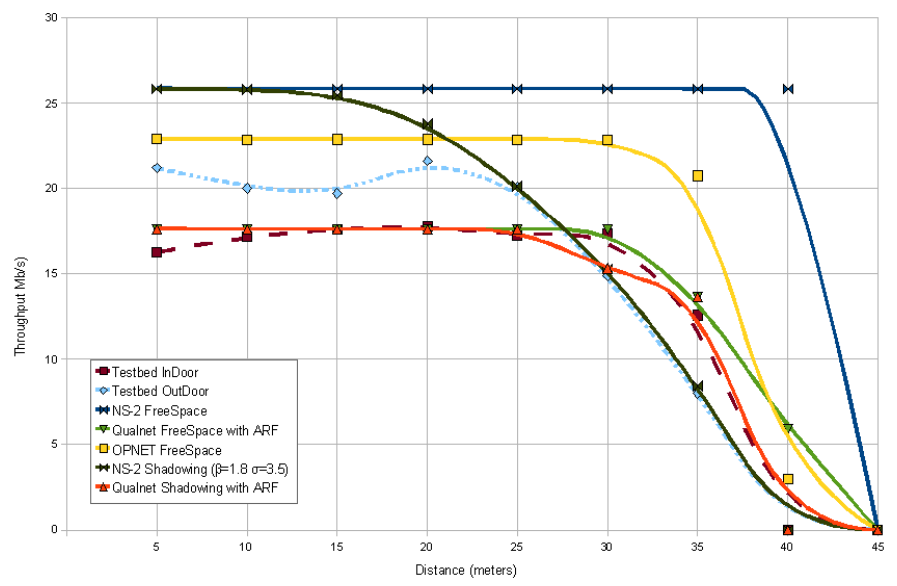

Figure 5. Average Throughput / Distance (Testbed compared to Simulators)

Figure 5 illustrates the obtained results from NS2, OPNET, QualNet simulators and the testbed. We remark that the simulation with ARF (QualNet) and shadowing model consideration (NS2 and QualNet) are close to testbed results in both cases indoor and outdoor environments. However, the parameter setting of this model is empirical and cannot be applied to other contexts.

\section{CONCLUSION}

In this work, we present a comparative study between results obtained from a real testbed and three usual network simulators (NS-2, Qualnet and OPNET). The main goal of this study is to evaluate the relevance of these simulators in indoor and outdoor environments. According to this study, we can point out that for the simulators, the choice of the PHY layer characteristics is predominant, particularly the propagation model and the associated parameters. The ARF procedure is only implemented in Qualnet which has also a very important role in the networks where the received power is variable according to the distance and the obstacles.

Concerning the dense multi-hop networks, the use in simulations of the free space or two ray ground models is not adapted to indoor contexts (majority of the cases in the $\mathrm{WiFi}$ deployment). However, we can use them only to validate routing algorithms without taking into account QoS or other parameters from the low layers.

In the future works, we plan to improve the lower layers of the network simulators in order to generate results which are closer to reality. To achieve this goal, we have to introduce ARF procedure and other propagation models with explicit parameters in order to reproduce a particular indoor environment.

\section{REFERENCES}

[1] Stuart Kurkowski, Tracy Camp, and Michael Colagrosso, "Manet simulation studies: the incredibles," SIGMOBILE Mob. Comput. Commun. Rev., vol. 9, no. 4, pp. 50-61, October 2005.

[2] David Cavin, Yoav Sasson, André Schiper, «On the Accuracy of MANETs Simulators », Proceedings of the second ACM international workshop on Principles of mobile computing, pp. 38 - 43, 2002.

[3] OPNET Modeler,

http://www.opnet.com/solutions/network_rd/modeler.html

[4] The Network Simulator NS-2, http://www.isi.edu/nsnam/ns/

[5] L. Bajaj, M. Takai , R. Ahuja, K. Tang , R. Bagrodia , M. Gerla, ," GloMoSim: A Scalable Network Simulation Environment", Technical Report 990027, UCLA Computer Science Department, 1999.

[6] Illya Stepanov, Daniel Herrscher, Kurt Rothermel, «On the Impact Propagation Models on MANETs Simulation Results», Proceedings of 7th IFIP International Conference on Mobile and Wireless Communication Networks (MWCN 2005), Marrakech, Morocco, September, 2005.

[7] AWE Communications, http://www.awe-communications.com

[8] G. F. Lucio, M. Paredes-Farrera, E. Jammeh, M. Fleury, M. J. Reed, "OPNET Modeler and ns-2 - Comparing the accuracy of network simulators for packet-level analysis using a network testbed", WSEAS Transactions on Computers, Vol. 2, No. 3, pp. 700-707, 2003.

[9] D. Cavin, Y. Sasson, A. Schiper, "On the accuracy of MANET simulators", Proceedings of the second ACM international workshop on Principles of mobile computing, pp. 38 - 43, Toulouse, France, 2002

[10] F. Kargl and Elmar Schoch, "Simulation of MANETs: a qualitative comparison between JiST/SWANS and ns-2", Proc. of the 1st international workshop on System evaluation for mobile platforms, International Conference On Mobile Systems, Applications And Services, pp. $41-46,2007$.

[11] Xu Su, Rajendra V. Boppana,"On the impact of noise on mobile ad hoc networks", Proceedings of the International conference on Wireless Communications and Mobile Computing (IWCMC'2007), pp. 208 - 213, 2007

[12] Michael Bredel, Martin Bergner, "On The Accuracy of IEEE 802.11g Wireless LAN Simulations Using OMNeT++", In Proc. of International Conference On Simulation Tools And Techniques For communications, Networks And Systems \& Workshops, 2009.

[13] P. Pablo Garrido, Manuel P. Malumbres, Carlos T. Calafate, "ns-2 vs. OPNET: a comparative study of the IEEE 802.11e technology on MANET environments", In Proc. of the 1st international conference on Simulation tools and techniques for communications, networks and systems \& workshops (SIMUTools), France, 2008.

[14] "MGEN: The Multi-Generator Toolset," June 2006, http://www.pf.itd.nrl.navy.mil/mgen/.

[15] NetPerf tool, http://www.netperf.org/netperf/

[16] QualNet, http://www.scalable-networks.com 